\title{
Look-Alike and Sound-Alike Medicines: Risks and 'Solutions'
}

Dr Lynne M Emmerton, Senior Lecturer*

Mariam FS Rizk, BPharm(Hons)

The University of Queensland

School of Pharmacy

St Lucia Qld 4072

Australia

* Corresponding author

Postal address: as above

Tel: $\quad+61-7-33461981$

Email: $\quad \underline{\text { 1.emmerton@ } @ \text { pharmacy.uq.edu.au }}$ 


\begin{abstract}
'Look-alike, sound-alike' medicines are associated with dispensing errors. This commentary aims to fuel discussion surrounding how drug name nomenclature and similar packaging between medicines can lead to selection errors, the need for enhanced approval systems for medicine names and packaging, and best practice 'solutions'. The literature reveals a number of environmental risks and human factors that can contribute to such errors. To contextualise these risks, we interviewed 13 quality and safety experts, psycholinguists, and hospital and community pharmacy practitioners in Australia, and commissioned a medical software industry expert to conceptualise electronic initiatives. Environmental factors contributing to such errors, identified through both the literature and interviews, include distractions during dispensing; workflow controls should minimise the 'human factors' element of errors. Technological solutions with some support, and yet recognised limitations, include font variations, automated alerts, barcode scanning and realtime reporting programmed into dispensing software; further development of these initiatives is recommended.
\end{abstract}

\title{
Introduction
}

Accuracy in the dispensing of medicines is vital for patient safety. A major contributor to dispensing errors is "look-alike and sound-alike" (LASA) medicines (Figure 1) ${ }^{1}$. Around one in four medication errors has been attributed to orthographic (look-alike) and phonetic (sound-alike) similarity between drug names and/or confusable packaging ${ }^{2-5}$. Of concern is that these errors have in common a human element, and are preventable through vigilance and understanding of their root cause.

In this commentary, we draw attention to risk factors and call for further research into riskreduction initiatives. Our review of the literature was supplemented with findings from semi-structured interviews with four clinical governance experts, four hospital pharmacy practitioners with experience in medicines safety initiatives, two community pharmacists, two psycholinguists and a quality and safety administrator in Australia. Participants were purposively selected to explore their experiences and opinions regarding a) risk situations in the selection of medicines during dispensing or administration processes and $b$ ) initiatives to reduce the identified risks. A representative from the Medical Software Industry Association reviewed the interview summary and provided expert opinion on the feasibility of technological 'solutions'. Presented below is a reflection that integrates the interview data (identifying the participants by their area of practice) with reports from published literature, where available, to provide an overview of the problems associated with LASA medicines.

\section{Risks Relating to Drug Nomenclature and Packaging}

A dominant drug name within a 'neighbourhood' of names familiar to an individual, and the 'density' of this neighbourhood (the number of competing similar names), can interfere with identification of the required medicine (clinical governance interviewee). A related issue is 'confirmation bias', whereby familiarity with a patient's history and the medicines commonly prescribed by certain doctors can result in erroneous assumptions (psycholinguistics). Confusion is more likely if the medicines appear in the same context (psycholinguistics). A notable example exists with 'Z-drugs' (the hypnotics zolpidem and 
zopiclone) marketed in close succession (community pharmacy). An added complication in Australia arises from the many generic 'brands' marketed (clinical governance); dispensers might wrongly assume that they know the brand name of the generic alternative (community pharmacy).

These insights have implications for approval of drug names in Australia. There is potential to automate screening to identify proposed names that are orthographically and/or phonetically similar to existing medicines, as implemented by United States Food and Drug Administration (clinical governance). 'Uniqueness' of medicine names can be improved by the distinguishing syllable(s) being at the start or middle of medicine names. Longer medicine names can increase the risk of confusion, yet the shorter the name, the greater the chance of similarity to existing names (psycholinguistics).

Risks for drug name confusion are confounded by the availability of different medicines in similar strengths, pack sizes, colours and dosage forms, and their use for similar conditions (clinical governance). Pharmacists face many medicines packaged in white bottles or boxes, unless different coloured caps or other distinguishing markers are used, and packaging updates might not be recognised (community pharmacy). Optimal design of manufacturers' labels has been explored in Denmark (www.e-types.dk/39267.9000/), with recommendations for font contrasts, legibility and layout; this appears to have merit for international application (quality and safety).

\section{Errors during Dispensing Processes}

Review of the international clinical literature and individual case reports reveals that misidentification of medicines may arise from the misreading of computer-printed or handwritten prescriptions or medication orders, mishearing of a spoken medicine name, wrong selection of similar-looking medicine names from lists in dispensing software, or wrong selection of a medicine from the shelf, either due to similar packaging or misidentification of the name $e^{6,7,9-11}$. Particular attention should be paid to selecting medicines from lists (community pharmacy); confusion may arise when selecting from lists if the eye focuses on the start and end of a medicine name, not recognising a distinguishing syllable mid-word (psycholinguistics).

Factors contributing to these errors include chaos and interruptions (community pharmacy, psycholinguistics). Such distractions lengthen the time that a medicine name must be held in one's working memory, to the critical point of "lose it or confuse it" (psycholinguistics). Best practice would dictate returning to the previous step if dispensing is interrupted, and separation of dispensing and checking processes (community pharmacy), perhaps utilising dispensing technicians, with pharmacists undertaking responsibility for the final check (medical software).

Pronunciation of the drug out loud may ensure effective reading of its name and retention in one's working memory (psycholinguistics). Similarly, verbal prescriptions should be repeated to the prescriber, including spelling out the medicine name ${ }^{8}$. People with difficulty pronouncing long medicine names are probably at a greater risk of confusing LASA medicines (psycholinguistics).

Locum pharmacists may be unfamiliar with the dispensing system in a pharmacy or with the dispensary layout, resulting in 'picking' errors particularly under time pressure 
(community pharmacy). Separation of confusable medicines on the shelf prompts the dispenser to search for the required medicine (community pharmacy). This is consistent with published recommendations ${ }^{8}$, although signposting of the shelf locations should be used strategically to avoid 'alert fatigue' (community pharmacy). In one hospital, LASA medicines have been identified with additional yellow labels under their regular labels. Other hospital pharmacy initiatives have been the arrangement of medicines by generic names, with laminated warning signs to mark commonly confused LASA medicines (hospital pharmacy). Practical suggestions reported in the literature also include limiting hospital formularies, eliminating abbreviations and encouraging patients to question physical changes in their medications ${ }^{12}$. The need for "system-based strategies" to prevent these errors has been recognised ${ }^{8,13}$, and there is potential for research at various levels to investigate the acceptability and outcomes of these initiatives.

Other environmental risks are inadequate lighting, illegible handwriting, and lack of familiarity with variations in generic and/or brand names of medicines and packaging, particularly small labels on ampoules or vials ${ }^{1,6-8}$. It is recommended that dispensing staff familiarise themselves with medicine names and packaging on an "internal" level, i.e. get to know the medicine and not just recognise its dominant features (psycholinguistics).

\section{Technological Solutions}

Four 'technological solutions' integrated into dispensing systems have been identified, with mixed support in the literature and amongst the interviewees:

1. Font variation. This involves manipulation of written text to highlight the distinguishing syllable(s) or characters between similar drug names, as in Tall Man lettering (Figure 2$)^{14}$. Research has involved limited numbers of subjects and experimental exercises in either field or laboratory conditions, and in some instances, using lay subjects, with equivocal error reduction but positive acceptability data ${ }^{11,15-17}$. Theoretically, Tall Man lettering should enhance dispensing accuracy (hospital pharmacy, clinical governance, quality and safety) and have an educative function ${ }^{17}$. However, concerns are that:

a. Mixed-case lettering may increase error rates if it interferes with the reading of a medicine name (psycholinguistics) or prolongs reading time (hospital pharmacy). This equates to "destroying" a word shape. ${ }^{7}$

b. If Tall Man lettering induces an upper-case first letter for a generic medicine, this may give the appearance of a brand name (psycholinguistics).

c. A number of medicines may not be adequately distinguished using Tall Man lettering (hospital pharmacy). An example is the 'triangulation' in representing Oxycontin ${ }^{\circledR}$ versus MS Contin ${ }^{\circledR}$ versus OxyNorm ${ }^{\circledR}$, oxycodone and Oxytrol ${ }^{\circledR}$ (clinical governance).

d. Without national guidelines, Tall Man lettering could be overused or used inconsistently (clinical governance, quality and safety). Restriction to a small percentage of confusable medicines, and at the point during dispensing where medicines are selected from lists (hospital pharmacy, medical software), were suggested.

e. Some flexibility would be warranted to allow dispensing staff to contribute actual and near-miss errors to the list (hospital pharmacy, medical software) ${ }^{6,18}$. 
Despite these concerns and lack of conclusive data, Tall Man lettering has potential for use in electronic selection menus, shelf labels and dispensing labels (clinical governance, medical software). This would be a relatively low-cost initiative, and a number of organisations are well positioned to collaboratively develop, manage and monitor this initiative (medical software).

2. Automated alerts during dispensing of LASA medicines. This is not a new concept, but further development is recommended. All major dispensing software in Australia incorporates warnings of various types, which originate from Australia's major pharmacy indemnity organisation, Pharmaceutical Defence Limited, in collaboration with software vendors. Some systems allow pharmacists to add alerts, and vary how the operator acknowledges the warnings (medical software). Mixed support has been reported. ${ }^{5,8,19,20}$ The key concern is "alert fatigue" (clinical governance, community pharmacy), so use should be strategic and guided by risk assessment (clinical governance), and there is potential for further manipulation of the alerts to optimise their effectiveness (medical software).

3. Barcode scanners to confirm drug selection against the medicine selected on the dispensing screen. ${ }^{8}$ This development has been generally accepted in community pharmacy in Australia, where financial incentives have been made available to purchase the hardware (medical software). Barcode scanning should theoretically eliminate most errors if used appropriately. However, reliance on scanning may merely "relocate" human factors problems rather than obviate them, and perpetuate prior errors. Manual checking procedures are still required to detect cases of erroneous data entry (community pharmacy, clinical governance). Further, there was concern that the use of barcode scanners may reduce the impact of other initiatives to reduce LASA risks (clinical governance).

4. Real-time reporting. Awareness of the risks of misidentification of medicines may be improved by case reporting by health professionals, with near-miss and error reporting incorporated electronically into dispensing software (medical software). Hospitals use electronic incident recording systems for documentation of LASA medicine errors (hospital pharmacy). In community pharmacy, software vendors release monthly updates, which could include LASA medicine alerts for printing (medical software).

\section{Discussion}

Many risks for LASA errors have origins beyond the practice environment (e.g. drug nomenclature and similar packaging of medicines), and ideally would be reduced during pre-marketing approvals of medicines. Accepting that some degree of similarity will always exist between drug names and between product packaging, we also draw attention to risk reduction at the practice level, where it should be noted that all errors have a 'human' element, such as making assumptions, failure to correctly hear or visually check, and distractions relating to fatigue and/or a busy work environment. These types of errors should not occur in ideal work practices. Human factors associated with errors were explored using psycholinguistics principles, revealing interesting phenomena regarding how the brain registers, processes and stores words (names of medicines) and images (pack shapes and characteristics), and how font variations and environmental influences (interruptions) can influence these processes. 
We recognise that the field of psycholinguistics is much more extensive than the insights reported here, and that there is potential to further involve this science in trials of word and packaging design. Further, the limited number of interviewees does not aim to be representative of the full spectrum of pharmacy practice, and merely serves to explore issues from a number of perspectives. It should be noted, though, that the majority of literature on this topic stems from individual case reports, so we believe that integrating the experiences of interviewees with the literature is a legitimate approach to expand this topic. Our focus has been on errors during dispensing. The numerous other stages of the medication cycle, from dispensing through to administration of doses, offer many avenues for research into risk-reduction strategies. Indeed, the need for further user studies involving healthcare professionals and patients has been recognised in the literature ${ }^{3}$.

Together, review of the literature, the series of key informant interviews and conceptualisation of electronic initiatives have given rise to a number of practice-based recommendations and technological 'solutions' that may be used individually or in a multifaceted approach to reduce risks associated with LASA medicines and work towards errorfree dispensing.

\section{Acknowledgements}

The authors gratefully acknowledge assistance from all pharmacists and drug safety and psycholinguistics experts who enthusiastically participated in this study and shared resources to contribute to this research.

\section{Funding}

This work was supported by the Pharmacy Research Trust, administered by the Pharmaceutical Society of Australia Queensland Branch Committee. MR was the recipient of a University of Queensland Summer Scholarship.

\section{References}

1. James KL, Barlow D, McArtney R, Hiom S, Roberts D, Whittlesea C. Incidence, type and causes of dispensing errors: a review of the literature. Int J Pharm Pract 2009; 17: 7-8.

2. daCosta P. Medication errors: what to do about look-alike/sound-alike drug names. Drug Topics 2009; 153: 52.

3. Lambert BL, Lin SJ, Chang KY, Gandhi SK. Similarity as a risk factor in drug-name confusion errors: the look-alike (orthographic) and sound-alike (phonetic) model. Med Care 1999; 37: 1214-25.

4. Joshi MC, Joshi HS, Tariq K, Ejaj A, Prayag S, Raju A. A prospective study of medication errors arising out of look-alike and sound-alike brand names confusion. Int $\mathbf{J}$ Risk Saf Med 2007; 19: 195-201.

5. daCosta P. Look-alike/sound-alike errors get more attention. Drug Topics 2009; 153: 52.

6. Hoffman JM, Proulx SM. Medication errors caused by confusion of drug names. Drug Saf 2003; 26: 445-52.

7. MHRA. Report to the committee on safety of medicines from the working group on labelling and packaging of medicines. UK: Medicines and Healthcare Products Regulatory Agency; 2001.

8. Tuohy N, Paparella S. Look-alike and sound-alike drugs: errors just waiting to happen. J Emerg Nurs 2005; 31: 569-71. 
9. The Joint Commission, USA. Sentinel event alert. Look-alike, sound-alike drug names: <www.jointcommission.org/SentinelEvents/SentinelEventAlert/sea_19.htm>.

10. Beyea SC. Confusing, look-alike, and sound-alike medications. Aorn J 2007; 86: 861-3.

11. Gerrett D, Gale A, Darker I, Filik R, Purdy K. Tall man lettering. Final report of the use of tall man lettering to minimise selection errors of medicine names in computer prescribing and dispensing systems. ITQ invitation reference no. ER-07-0612. UK:

National Health Service; 2009.

12. Sandhu G. Anticancer medication safety. J Pharm Pract Res 2011, 41(1): 74

13. Reason J. Human error: models and management. BMJ 2000; 320: 768-70.

14. Pettersson R. Attention: an information design perspective. Design Perspect 2001; 2: 114-30.

15. Filik R, Purdy K, Gale A, Gerrett D. Labeling of medicines and patient safety: evaluating methods of reducing drug name confusion. Hum Factors 2006; 48: 39-47.

16. Filik R, Purdy K, Gale A, Gerrett D. Drug name confusion: evaluating the effectiveness of capital ("tall man") letters using eye movement data. Soc Sci Med 2004; 59: 2597-601.

17. Van de Vreede M, McRae A, Wiseman M, Dooley MJ. Successful introduction of Tallman letters to reduce medication selection errors in a hospital network. J Pharm Pract Res 2008; 38: 263-6.

18. Gabriele $\mathrm{S}$. The role of typography in differentiating look-alike/sound-alike drug names. Healthc Q 2006; 9: 88-95.

19. Thompson CA. USP says thousands of drug names look or sound alike. Am J Health Syst Pharm 2008; 65: 386-8.

20. Fleming H. Computer alert may help prevent sound-alike drug errors. Drug Topics 1999; 143: 1. 


\section{Figure 1: Examples of LASA Medicines}

\begin{tabular}{|ll}
\hline Losec (omeprazole) & Prozac (fluoxetine) \\
Aropax (paroxetine) & Aratac (amiodarone) \\
Zocor (simvastatin) & Zoton (lansoprazole) \\
Mogadon (nitrazepam) & Maxolon (metoclopramide) \\
Xanax (alprozolam) & Zantac (ranitidine) \\
Diflucan (fluconazole) & Diprivan (propofol) \\
Carafate (sucralfate) & Caltrate (calcium) \\
\hline
\end{tabular}

Figure 1: Typographic Variations (adapted from Gabriele ${ }^{12}$ )

\begin{tabular}{|lll|} 
Contrast & Hydroxyzine & Hydralazine \\
Weight & Hydroxyzine & Hydralazine \\
Case (Tallman) & HydrOXYzine & HydrALAzine \\
\hline
\end{tabular}

